\title{
AN INTELLIGENT REAL-TIME WIRELESS SENSOR NETWORK TRACKING SYSTEM FOR MONITORING RHINOS AND ELEPHANTS IN TANZANIA NATIONAL PARKS: A REVIEW
}

\author{
Erick Alphonce Massawe ${ }^{1}$, Michael Kisangiri ${ }^{1}$, Shubi Kaijage ${ }^{1}$ and \\ Padmanabhan Seshaiyer ${ }^{2}$ \\ ${ }^{1}$ Department of Electronics and Telecommunication Engineering \\ Nelson Mandela African Institution of Science and Technology, NM-AIST \\ Arusha, Tanzania \\ ${ }^{2}$ Department of Mathematical Sciences, College of Science George Mason University \\ Virginia, United states of America
}

\begin{abstract}
The advancement of wireless sensor networks yields a variety of wireless sensor network for wildlife tracking. One typical application for wireless sensor networks is in animal tracking and monitoring in wildlife environments. A significant number of studies have been done in tracking animals with sensor networks. However from the recent literature it is observed that there is no much study has been done on an intelligent real time sensor network that is capable to alerting the rangers an incidence of animal poaching before it happened. In this paper an intelligent wireless sensor system for tracking and monitoring rhinos and elephants is proposed.
\end{abstract}

\section{KEYWORDS}

Wireless sensor network, poaching, rhinos, elephants, monitoring, tracking

\section{INTRODUCTION}

A Wireless Sensor Networks (WSN) is defined as a network of wireless devices, called as nodes, which sense given objects or entities and communicate the sensed data through wireless links. The data are transmitted via a single hop or multi-hops, to a control center, which can be connected to other networks, for example GSM. A wireless sensor node consists of one or more sensors for sensing physical variables, main processing unit (a microcontroller or low-power consuming processor), analog-to-digital converter (ADC), flash memory, and RF transceiver[13]. Wireless sensor networks have recently come into prominence because of its potential in revolutionizing many aspects of the economy and day to day life from environmental monitoring, to the health care industry, manufacturing etc. Sensors are often deployed in constrained environments, such as rainforests, mountains or construction sites, for monitoring or detecting particular events [4-6]. 
International Journal of Advanced Smart Sensor Network Systems (IJASSN), Vol 7, No.4, October 2017

\subsection{BACKGROUND OF PROBLEM}

Poaching decimated African elephant populations from 1.3 million to 500,000 individuals in the eight years between 1979 and 1987. This prompted the Convention on International Trade in Endangered Species (CITES) to list the African elephant as an endangered species in 1989. Despite these protections, a higher percentage of Africa's remaining elephants are now being killed than ever before[7]. Wildlife groups estimate that 10,000 to 25,000 elephants are killed in Tanzania each year for their ivory tusks and the number of elephants in southern Tanzania has fallen by more than half. Also, they estimate that as many as 50,000 elephants are currently being killed annually based on the 46.5 tons of African elephant ivory seized in 2011. Assuming approximately 400,000 elephants remaining in Africa, African elephants could be virtually extinct in the next decade[8].

The world is dealing with this unprecedented spike in illegal wildlife trade, threatening to overturn decades of conservation and development gains. As both populations and economies have grown in East Asia, the demand for wildlife products has surged, sending the black market price of ivory and rhino horn. The recent rise in wildlife product prices has been met by the increased involvement of more organized, better funded, and better armed criminal and terrorist networks, and even militias, compounding the challenges faced by those charged with protecting the wildlife. Wildlife products can easily be converted into cash and used to purchase weapons and fund violent campaigns, and have become a substantial source of income for terrorist organizations in Africa[9]. Asian demand for rhino horn has set a historic price for rhino horn powder - over $\$ 30,000$ per pound, making it more valuable than gold and cocaine.

Rhino poaching rates in Africa have risen since the beginning of the decade. About 500 rhinos a year are poached in South Africa alone, despite increased surveillance efforts in South African nature reserves, setting a new national record of rhino killings[9].

In 2011, elephant poaching levels in Africa were at their highest since international monitors began keeping detailed records in 2002, and recorded ivory seizures are at their highest levels since 1989. Estimates from the first half of 2012 provide little reason to think the trend will slow down any time soon. In year 2013 nearly 450 elephants were slaughtered in Cameroon's National Park, representing close to $10 \%$ of that country's remaining elephant population[9].

Illegal wildlife trade, driven by high profit margins and aided by poor governance and weak law enforcement efforts, has boomed, just as other Chinese investments in Africa have grown increasingly active. Wildlife agents, customs officials, and government leaders are being paid off with what is viewed as a well-organized mafia moving animal parts of Africa to Asia. Toothless laws, corruption, weak judicial systems, and light punishments allow criminal networks to thrive on wildlife trade with little regard to risk or consequence[9].

The extreme poverty of many African communities induces their complicity in African-based, Asian-run poaching networks. The demand for ivory has surged to the point that the tusks of a single adult elephant can be worth more than 10 times the average annual income in many African countries[9]. 
International Journal of Advanced Smart Sensor Network Systems (IJASSN), Vol 7, No.4, October 2017

\section{RELATED WORK}

This research suggest the use of animal attached with certain type of sensors and form an intelligent tracking and monitoring system that can be able to send information to the rangers when the animal is on panic condition or horns removed. Many scientific studies have put more efforts on development of systems that can only report to the rangers after the incidence of poaching had happened. Therefore both the use of sensors to form an intelligent tracking and monitoring of rhinos and elephants, existing anti-poaching systems and animal tracking and monitoring systems have to be investigated.

One of the recent rhinos and elephants ant-poaching tools used in Africa is Remotely Piloted Aircraft Systems. In this research conducted by Department of Sensor Science Technology, Council for Scientific and Industrial Research (CSIR) in collaboration with Centre for Wildlife Management, University of Pretoria, South Africa. The authors proposed the use of Remotely Piloted Aircraft System (RPAS) as a Rhinoceros Anti-Poaching Tool in Africa[10]

Remotely Piloted Aircraft Systems (RPAS), sometimes also referred as Unmanned Aerial Vehicles (UAVs), Unmanned Aerial Systems (UASs) or drones (the ones for military purposes), are aircrafts (fixed or rotary wings) that are equipped with cameras and or other sensors and can be sent (using manual, semiautomatic or automatic control) to a destination to gather information. These aircrafts act like an "eye in the sky" with the operator at the ground control station receiving data or sending orders to the aerial platform[11].

RPAS have been used for locating "enemies" in military applications for the last 20 years[12], and more recently they have started to play a role in many civilian tasks, including wildlife monitoring. RPAS research describe the use of a small low cost RPAS equipped with three different types of cameras to test their ability to support rhinoceros anti-poaching tasks in cooperation with a specialized security company working in the KwaZulu- Natal province of South Africa. They performed several flights in order to test the technical capabilities of the system to detect rhinoceros, to reveal simulated poachers and to do fence surveillance.

They evaluated the effectiveness of the system at different altitudes and times of the day and night, and over the two main habitat types in the area: open grassland and forest. Considering the most common modus operandi of poachers, they analyzed the aspects that affect remotely piloted aircraft's integration in anti-poaching operations. It perform poorly during the night, rain seasons, wind greater than $15 \mathrm{~km} / \mathrm{h}$, areas with high humidity, thick forest, populated areas and high altitude areas[10].

Most African countries like Tanzania have poor economy and the governments cannot be able to implement such system. The price for single RPAS equipment bought from Spain was 13,750€. Also, as a reference, the system they used has performed more than 500 flights with an approximate total investment of 14,000 $€$ [11]. This is the huge amount of money for a country like Tanzania to invest in only one sector while it is equivalent to the annual budget amount of a sensitive ministry and therefore it is not cost effective for Tanzania to employ it despite the fact that RPAS has contributed valuable and respectful thoughts in the field.

Another ant-poaching research has been conducted in Kenya with the aid of World Wild life Fund (WWF). The study involve deployment of specialized rhino horn tracking systems combined with forensic DNA technology which allow for 100 percent traceability of every rhino 
horn and live animal within Kenya. In the DNA study for anti-poaching involves implanting microchips into the horn of every rhinoceros in Kenya in a bid to keep the dwindling population safe from the ever-increasing presence of poachers. The microchips in the horns of rhinos may deter poachers from trying to smuggle the contraband out of Kenya. When a rhino is killed and the horn is hacked off and taken away, if this horn is confiscated and the microchip tag can be identified, it can be tracked back to a poached animal and it can actually show and prove that this was a poaching incident[13].

However, the approach presented in this research is to prevent poaching incidents before it happens and or while it is happening, which is worth enough than waiting a problem to happen and capture the poachers after they have already made poaching incident.

Another important study has been presented by Jamali Firmat Banzi, masters Student School of electronics engineering, Tianjin University of technology and education. The main idea presented in his paper involve employing a modern and a sophisticated technology in which poachers will be left behind and being netted easily there by eliminating Poaching activities. The idea utilize animals themselves with sensors as mobile biological sensors (MBS) mounted with sensor fusion (having visual infrared camera and GPS) that transmits the location of MBS, access points for wireless communication and a central computer system which classifies animal actions.

The system proposes three different actions of responses, firstly: access points continuously receive data about the animals' location using GPS at certain time intervals and the gathered data is then classified and checked to see if there is a sudden movement (panic) of the animal groups: this action is called animal behavior classification (ABC). The second action can be called visualization where by different image processing techniques of the obtained images surrounding an animal group are performed and therefore provide an ample assistance in understanding what makes a sudden movement of the animal group. The last action is to send messages to the game ranger's cellular phones about the panic of animals and the location through GSM network[14].

This proposed system has many drawbacks and is not efficient due to the following; For example, if the animal with the camera moved away from the group it is not possible to know what will happen. Rhinos and elephants not necessarily move in groups, and for this case, those animals which will be working along will not be protected. The sending of the images needs a network with high bandwidth so as to send the data with less delaying. It's very difficult to take pictures in dense forest and populated areas. Also the camera consumes much power; hence the cameras that have batteries with long life span are expensive and needs to be charged frequently.

Another study conducted in[15]. Remote monitoring of animal behavior in the environment can assist in managing both the animal and its environmental impact. GPS collars which record animal locations with high temporal frequency allow researchers to monitor both animal behavior and interactions with the environment. These ground-based sensors can be combined with remotely-sensed satellite images to understand animal-landscape interactions. The key to combining these technologies is communication methods such as wireless sensor networks (WSNs). The author explores this concept using a case-study from an extensive cattle enterprise in northern Australia and demonstrates the potential for combining GPS collars and satellite images in a WSN to monitor behavioral preferences and social behavior of cattle[15]. However this concept cannot be implemented for poaching monitoring since satellite is very expensive and a specific satellite should be positioned to monitor the specific area. 
Another paper was presented by Ravi Bagree et.al suggested the use of Wireless Sensor Network (WSN) in combination with image sensors opens plethora for wildlife tracking. It provides a glimpse into previously unseen, remote and inaccessible world of some of the most endangered species on earth. TigerCENSE is such an attempt to put sensor network technology in conserving one of the rarest and most elusive big cat species. The node, triggered by the Passive Infrared (PIR) sensor, captures the image of a tiger using a CMOS image sensor and stores it in an external memory chip. To avoid any disturbance to the animals, the node uses an Infrared (IR) flash, instead of white flash, to illuminate the target at night. The stored images get transferred to the base station via radio transceiver. This is transferred to the database server through Internet links for analysis by wildlife researchers. A solar energy harvesting system for recharging node's batteries is being added to avoid frequent human visit to change the batteries, making it highly non-intrusive system[16].

This technology if applied for the rhinos and elephants monitoring it will not work with good efficiency due to the following reasons; many cameras will be required throughout the national park, processing of image data requires enough bandwidth and also the cameras do not work properly during night hours and in thick forest.

In addition to these, there is another important research which is similar to the study presented in this paper. Using biometric sensors to monitor the animal body parameters is not a new idea, but it has been limited to a few of incident types. For example, Lei Zhang on his paper he described a distributed system for mad-cow monitoring and tracking. In his paper he proposed the use of the tiny sensor device which is able to detect virus, read the current level of body temperature, measure the heart-beating rate and blood pressure for each cow. He proposed Base stations to be used for data processing since it has higher processing capability and more energy source, such as PDAs. And they are installed in the barn, stones or trees in the grass field, in the transportation trucks and slaughtering houses. All base stations, grouped into a local monitoring network, can forward information to a central base station which connects with the Internet and can save the data information or forward them to the control center. The proposed system can provides disease detection and safety processing monitoring [17].

\section{Motivation}

Collectively the previously stated methods and others which are similar in regard to animals monitoring and tracking may be successful in their own right, but, applying these into a single homogenous system would have significant return. In addition, this paper offers a solution to problems stemming from using drones and Satellites in detecting animal behavior or Poaching prevention. Using satellites focused only on National parks causes many extra costs and, of course it requires a satellite to exist. Also using drones for poaching prevention has resulted many problems including mistakenly attack and issues of human right breaching. Using intelligent wireless sensor network tracking and monitoring system will report the poaching incident at the time it happens. The proposed sensor system has built in measures to use animal panic behaviors and horns synchronizations to Poaching incident, and show the location though GPS. Furthermore, this system may assist monitoring normal animal's death, and understanding animal's group behaviors. 


\subsection{AN INTELLIGENT TRACKING AND MONITORING SYSTEM}

This research paper proposes the use of an intelligent tracking monitoring system. An appropriate endangers animals are chosen in this case elephants and rhinos and attached with appropriate sensors. The preferred part of the body, tusk and horns of elephant and rhino are attached with sensors which will be synchronized to each other and to the gateway wireless network. The part of the body, horns and tusk must be carefully chosen such that it will not disturb an animal and give wrong result because of unnecessary movements resulting from scaring attached sensors. Also the position of the sensors must be in such a way that they will also be able to send information to the gateway wireless network.

\subsection{FIELD EXPERIENCE ON ELEPHANTS COLlARING PROJECT AT RUAHA NATIONAL PARK}

On October 2016 we were invited by World Elephant Centre (WEC) for elephants collaring program at Ruaha national park. The main objectives of collaring the elephants was to monitor their seasonal movements and distribution patterns map their habitats and routes within and outside the reserves provide information to enhance their protection through rangers patrols and land use planning. The elephant collar to be fitted on the neck of an elephant consists of satellite GPS, temperature sensor and a powerful battery which can run for 3-5 years. The GPS on the collar sends information of the location and activity pattern of the elephant on specified time intervals such as every 20,30 minutes etc. such information will be useful in mapping the activity pattern of the elephant as part of its monitoring. This is illustrated in Figure 1 with one of the coauthors fromTanzania with the GPS collars.

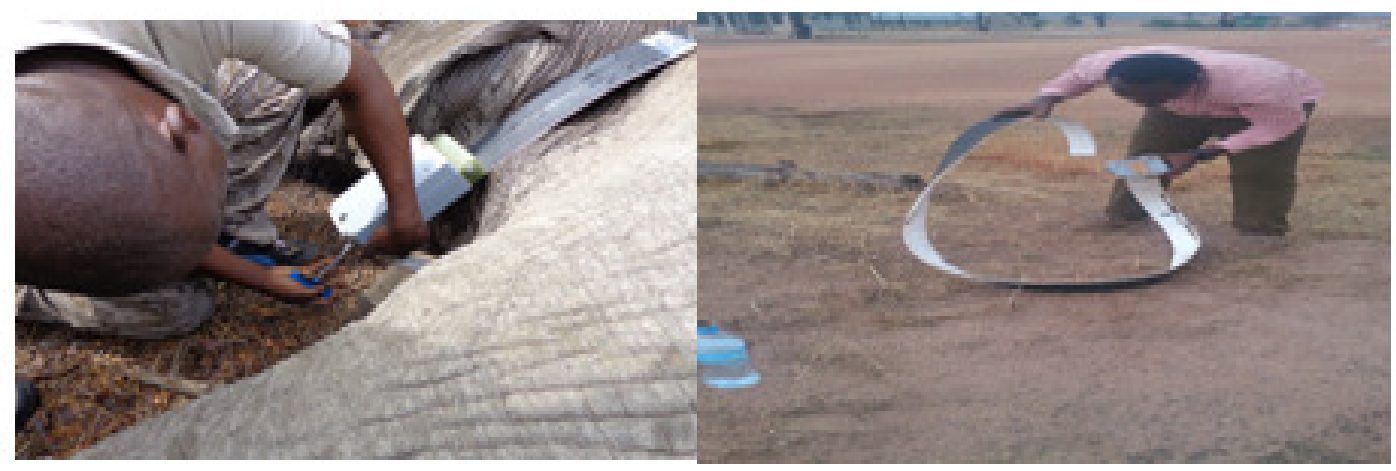

Figure1. Elephants Collaring at Ruaha National Park

\section{PROPOSED SYSTEM INFRASTRUCTURE}

The devices essential to the system includes the following:

A. Communication channel: The best method proposed in this paper is the use of access points as this method is cost effective. Access points are used to collect movement data from sensors attached to elephants (MBS) and send them to Central computer. To succeed these GSM base stations such as those of telecommunications companies like TIGO, VODACOM and AIRTEL can be used. Also high voltage poles, tall massive trees and watchtowers are possible access points. However satellite based systems can also be 
applied; employing satellite system is fairly complex and costly as it requires a satellite to focus on specific area only such as national parks only.

B. A central Computer system: This device must be trained with neural network, the device will classify data received continuously from the animal body sensors and GPS via access point and suggest if its normal panic (harassment) or it's a panic caused by a dangerous action like poaching.

C. Sensors network: The sensor network comprises with smart sensors attached into the horns and body of the animal. Zigbee wireless sensors will be used to connect the signals from the synchronized horns to the collar tag. Portable GSM modem/ Zigbee will be the default gateway from the collar tag, which will send the signal to the sensor nodes access points and then to base station. All the sensors will be powered by small solar panels with Lithium ion battery, which will be attached to the horns and the collar tag. Also Piezoelectric will be used as the backup charging mechanism in case of the solar power failure.

D. Cellular smartphone: Mobile application software will be designed for receiving a message about the panic of animals and the location where that panic is taking place. It is recommended that the device must be owned by game rangers at work who will be ready to take action whenever they receive a message, however the information received in the cellular phone must be synchronized with those from a central computer system for reliability.

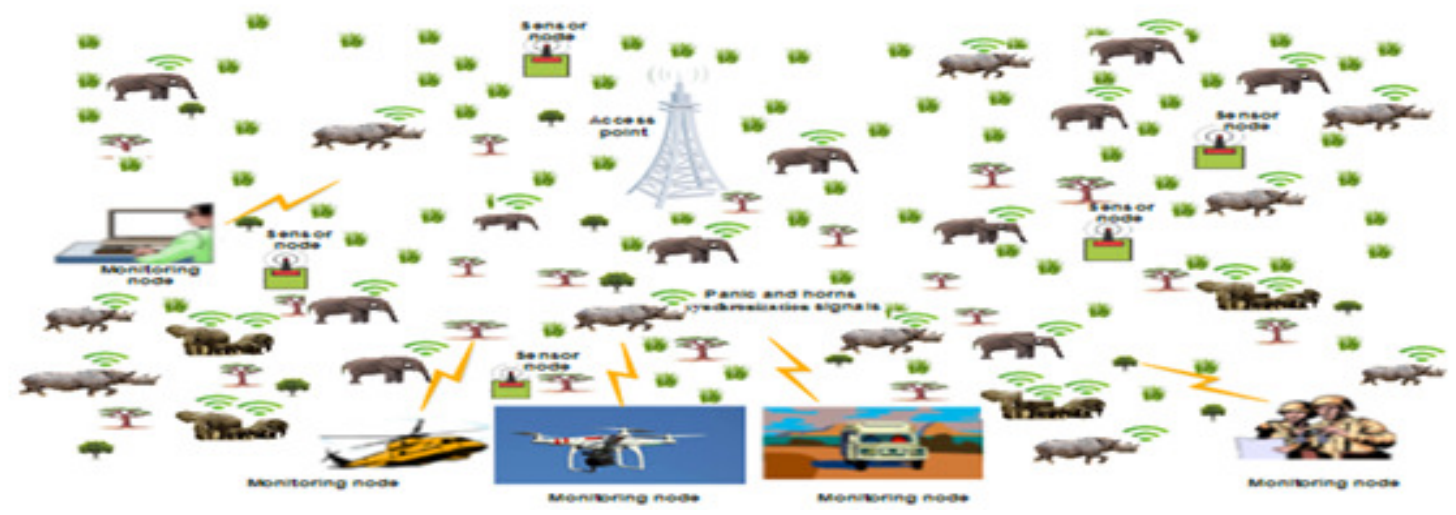

Figure 2. Poaching preventation system infrastructure

\subsection{SYSTEM OPERATION}

The system works as follow: Access point receives sensors location continuously and sends it to central computer where it stores it in a database. This computer is fed with a specific algorithm of Artificial intelligent. A classifier indexed to the database will continuously check the database to determine any abnormalities on sensors values. Using artificial intelligent tool a classifier will attempt to determine whether or not there are abnormalities on animal bodies parameters, horns line of sight synchronization and voice or sound compared to usual learned behavior of the animal. If a sudden panic of animals occurs an abrupt change in the graph of a classifier in the central computer occurs, this shows a potential incident and the system respond by first rises an alarm, secondly displaying the current location and time using GPS. 
Furthermore a system will send a short message (SMS) to the game rangers and drones through a GSM network to draw their attention on the suspected area. If immediate measures are taken by the game rangers poachers will be easily arrested and poaching will be eliminated in this way.

\section{DISCUSSION}

The proposed ant-poaching intelligent system by this paper uses sensors. In order to determine whether to apply this system in the particular parks such as Tanzania National parks, it may be crucial importance to look at some advantages and disadvantages of the system. Amongst advantages of the system are the system is very convenient and can be easily adopted to current anti-poaching systems, the system is cost effective as sensors are cheap and can be integrated to available infrastructure, Applying this system will reveal more knowledge on animal behavior, also the system can be applied for other purpose such as comprehensive animal death.As stated above the system may have some drawbacks such as: It is not easy to capture these dangerous animals like elephant and attach with sensors and hence need trained personnel. Also the use of battery brings about many problems such as pollution and extra radiation.

\section{CONCLUSION AND FUTURE WORK}

This research paper presents an intelligent animal tracking and monitoring system, and therefore it can be applied to any dangered species at a particular time. However the system doesn't come to replace the current anti-poaching initiatives, but rather to combine the effort in order to get rid of poaching incidence. This system could prevent potentially serious Poaching activities occurring in different parts of Africa particularly Tanzania. It is expected that if Tanzania National Parks (TANAPA) employ this sophisticated technology to the current worse situation of poaching activities will be of great advantage to TANAPA as it is reliable and will reduce the economic burden of investing too much on Anti- poaching. At this stage the system is under designing and prototyping. Even though it is beyond this project application of wireless technology and computer vision will dramatically improve the system. Lastly new sensors with improved capacity and robustness can be applied to the system. It is noticed that the life of important animal species is threatened today due to poaching, and if affirmative actions are not enforced, the loss of certain species which will lead to dramatic ecological imbalance may occur. This paper aims at presenting an alternative way to fight Poaching using sophisticated technology which seems to be effective and cost-effective to employ and use it.

\section{REFERENCES}

[1] Y. Chen and X. Wang, "Design of Distributed Intelligent physiological parameter Monitor System Based on Wireless Communication Technology," in Future BioMedical Information Engineering, 2008. FBIE'08. International Seminar on, 2008, pp. 403-406.

[2] J. S. Choi and M. Zhou, "Recent advances in wireless sensor networks for health monitoring," Int. J. Intell. Control Syst, vol. 14, pp. 49-58, 2010.

[3] A. T. H. Barth, Mark A Powell, HC Lach, John, "TEMPO 3.1: A body area sensor network platform for continuous movement assessment," in Wearable and Implantable Body Sensor Networks, 2009. BSN 2009. Sixth International Workshop on, 2009, pp. 71-76. 
International Journal of Advanced Smart Sensor Network Systems (IJASSN), Vol 7, No.4, October 2017

[4] Elson J. \& Estrin D, "Sensor networks: A bridge to the physical world," Norwell, MA: Kluwer Academic Publishers, 2004.

[5] C. D. Estrin D., Pister K., \& Sukhatme G.,, "Connecting the physical world with pervasive networks," IEEE Pervasive Computing, 2002.

[6] C. A. Ganesan D., Ye W., Yu Y., Zhao J., and Estrin D, "Networking Issues in Wireless Sensor Networks," Journal of Parallel and Distributed Computing (JPDC), 2004.

[7] University of Washington. (2013). Center for Conservation Biology. Available: http://conservationbiology.uw.edu/research-programs/tracking-poached-ivory/

[8] A. K. Sen. (2013) Tanzania's storied wildlife reserves could soon get a watchful, winged inhabitant: U.S. drones. The Washington Times

[9] ICCF.(2013). The African Poaching Crisis. Available:

http://iccfoundation.us/index.php?option=com_content $\&$ view=article $\&$ id=445\&Itemid=367

[10] R. S. Margarita Mulero-Pa' zma'ny, L. D. van Essen, Juan J. Negro,Tyrell Sassen, "Remotely Piloted Aircraft Systems as a Rhinoceros Anti-Poaching Tool in Africa," PLOS ONE, vol. volume 9, January 2014.

[11] J. J. N. m. Airam Rodríguez, Mara Mulero, Carlos Rodríguez, Jesús Hernández-Pliego, Javier Bustamante, "The Eye in the Sky: Combined Use of Unmanned Aerial Systems and GPS Data Loggers for Ecological Research and Conservation of Small Birds," PLOS ONE, vol. volume 10, December 11, 2012.

[12] M. Zenko, "Reforming U.S. Drone Strike Policies," January 2013.

[13] W. World Wildlife Fund. (16 october 2013) Microchip to protects Rhino in Kenya. Available: www.panda.org/

[14] J. F. Banzi, "A Sensor Based Anti-Poaching System in Tanzania National Parks," International Journal of Scientific and Research Publications, vol. Volume 4, 4, April 2014.

[15] D. L. S. Rebecca N. Handcock, Greg J. Bishop-Hurley, Kym P. Patison, Tim Wark, Philip Valencia, Peter Corke, Christopher J. O’Neill "Monitoring Animal Behaviour and Environmental Interactions Using Wireless Sensor Networks, GPS Collars and Satellite Remote Sensing," OPEN ACCESS, vol. Volume 9, 13 May 2009.

[16] R. J. Bagree, Vishwas Raj Kumar, Aman Ranjan, Prabhat, "TigerCENSE: wireless image sensor network to monitor tiger movement," in Real-World Wireless Sensor Networks, ed: Springer, 2010, pp. 13-24.

[17] L. L. Zhang, Alvin S Song, Hui Zheng, Xinliang, "A Distributed SYSTEM FOR MAD-COW DISEASE MONITORING AND TRACKING," International Journal of UbiComp, vol. 1, 2010. 


\section{AUTHORS}

Erick A. Massawe is currently a $\mathrm{PhD}$ student in Information and Communication Science and Engineering at Nelson Mandela African Institution of Science and Technology (NM-AIST) majoring in Electronics and Telecommunication Engineering. He received MSc degree in Information and Communication Science and Engineering at NM-AIST majoring in Telecommunications Systems Engineering in December 2013. He also received BE (Hons) degree in Electronics and Communication Engineering from St Joseph University in Tanzania (SJUIT) in November 2010. He worked with Kilimanjaro International Institute of Telecommunication, Electronics and Computer (KIITEC) as the Telecommunication and Electronics Instructor in October 2010, then as Educational Director.

His research interests include Electronics, Network Design, Biomedical Engineering, Data communication, Data Transmission and Wireless sensor networks. He is a member of the institution of Engineers Tanzania. He is also a member of IEEE registered as a Graduate Student.

Dr. Kisangiri Michael received his $\mathrm{PhD}$ (Telecommunication Engineering) from Wroclaw University of Technology Poland, Institute of Telecommunication and Acoustics in December 2008. In April 2002 he graduated from the same University, Master of Science in Telecommunication Engineering (Department of Radio communication) with specialization in Mobile Communication. He has been working with

Dar es Salaam Institute of Technology (D.I.T) in the department of Electronics and Telecommunication Engineering since October 2002 as Assistant Lecturer to November 2008, then as a Lecturer to November 2011.

In December 2011, He joined Nelson Mandela African Institute of Science and Technology (NM-AIST) in the College of Computational and Communication Science and Engineering (CoCSE) as a Lecturer and then promoted to Senior Lecturer 2016. His area of interests includes evolutionary computation in Telecommunication networks; Antenna design and Triangular mesh modeling.

Dr. Shubi Kaijage received his PhD on Optical fiber communication 2014. He has been working with THz Technical Research Center (TTRC) of Shenzhen University as research fellow from September 2011 to January 2014, Fiber Optics Lab, University of the Ryukyus as a research assistant from April 2008 to March 2011 and Davis and Shirtliff Co. Ltd as senior sales Engineer from March 2001 February 2005.

In February 2014, He joined Nelson Mandela African Institute of Science and Technology (NM-AIST) in the College of Computational and Communication Science and Engineering (CoCSE) as a Lecturer and then promoted to Head of department of Electronics and Telecommunication Engineering 2015. His area of interests includes Optical fiber communications, Telecommunication systems and
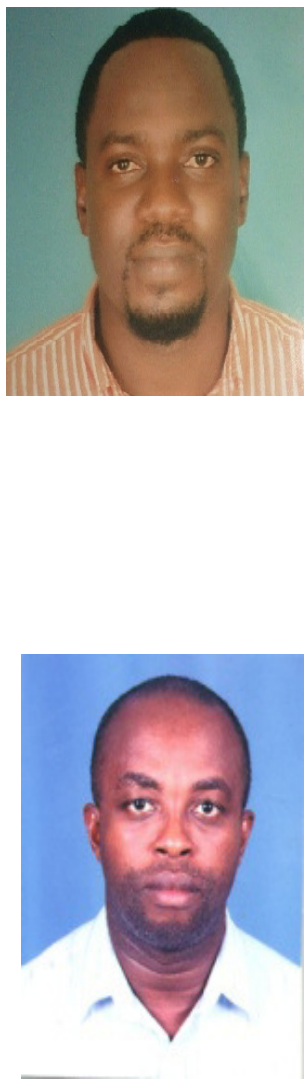
sensor networks. 
International Journal of Advanced Smart Sensor Network Systems (IJASSN), Vol 7, No.4, October 2017

Prof. Padmanabhan Seshaiyer received a B.E. (Hons) degree in Electrical and Electronics Engineering and a M.Sc (Hons) degree in Mathematics from Birla Institute of Science and Technology, India in 1994. Following that he received a Ph.D. in Applied Mathematics from University of Maryland, Baltimore County in 1998. He worked as a Post-doctoral research associate in the bioengineering program at Texas A\&M University from 1998-2000. He served as a faculty of Mathematics from 2000-2007 at Texas Tech University where he was a tenured member. Since 2007 he has served as a tenured faculty member of Mathematical Sciences at George Mason University, USA and also holds an adjunct faculty appointment at the Nelson Mandela - AIST since 2012.

His interests include mathematical and computer modeling, advanced scientific computing and STEM education.

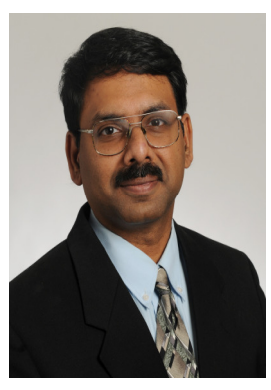

